\title{
DAMPING RATES OF THE SRRC STORAGE RING
}

\author{
K.T. Hsu, C.C. Kuo, W.K. Lau, W.T. Weng* \\ Synchrotron Radiation Research Center
}

No 1. R\&D Rd VI, Hsinchu Science-Based Industrial Park, Hsinchu, Taiwan, R.O.C.

\section{Abstract}

The SRRC storage ring is a low emittance synchrotron radiation machine with nominal operation energy $1.3 \mathrm{GeV}$. The design damping time due to synchrotron radiation is $10.7,14.4,8.7 \mathrm{~ms}$ for the horizontal, vertical and longitudinal plane, respectively. We measured the real machine damping time as a function of bunch current, chromaticity, etc. To damp the transverse beam instability, especially in the vertical plane, we need to increase chromaticity to large positive value. The damping rates are much larger than the design values. Landau damping contribution in the longitudinal plane is quite large, especially in the multibunch mode. The estimated synchrotron tune spread from the Landau damping is in agreement with the measured coherent longitudinal coupled bunch oscillation amplitude.

\section{INTRODUCTION}

In the synchrotron storage ring at SRRC, we have observed transverse and longitudinal beam instabilities. $[1,2]$ It is imperative that the machine provide a stable, high brilliant synchrotron light source to user community so that the users could take full advantage of such a powerful facility. To this end, several measures have been taken to suppress the instabilities.

In the transverse planes, the beam oscillations were attributed to the beam-ion interaction and the most effective and convenient way up-to-date is either to increase the chromaticities by raising the excitation level of the sextupole magnets or to employ the transverse damping system. With overcorrected chromaticities, the major damping sources include head-tail damping and Landau damping. It was found that the growth time of the beam-ion instability could be as fast as a few ms under normal operation condition, i.e., with $200 \mathrm{~mA}$ stored beam and 1.5 nTorr vacuum condition. Therefore, typical chromaticity setting is more than 3 in the vertical plane. The increase of the nonlinear field strength in the sextupole magnets not only reduce the dynamic aperture but also cause the injection difficulty. Moreover, it alters the beam orbit and increases the emittance coupling in the transverse planes. Despite of such disadvantages, it is the best way to suppress this instability before other promising cures are applied in the near future, e.g., transverse damping system.

In the longitudinal plane, the higher order modes (HOM) of the if cavities are responsible for the coupled bunch instabilities. These instabilities have to be eliminated to justify the low emittance, high brightness lattice design.

\footnotetext{
"Permanent address: AGS Department, BNL Upton, NY, USA.
} Work partially performed under the ausplces the U.S. Dept. of Energy.
Therefore, we plan to control cavity temperature so that the HOM can be removed away from beam resonance. We also consider to implement a longitudinal damping system.

This article describes the observation of the damping phenomena of the kicked beam in the transverse and longitudinal plane, respectively. The decoherence and recoherence of the kicked beam centroid are discussed. A preliminary transverse damping system in test is briefly described.

\section{TRANSVERSE DAMPING}

A high current single bunch beam was populated and excited with If knock-out method in both planes, either simultaneously or independently. The beam signals from the pick-up electrodes were analyzed with spectrum analyzer. The amplitude of the horizontal and vertical betatron sidebands was displayed and recorded in a fast oscilloscope. The decay pattern then was analyzed off-line for different ring parameter settings. Fig. 1 shows a typical decay pattern with different chromaticity.

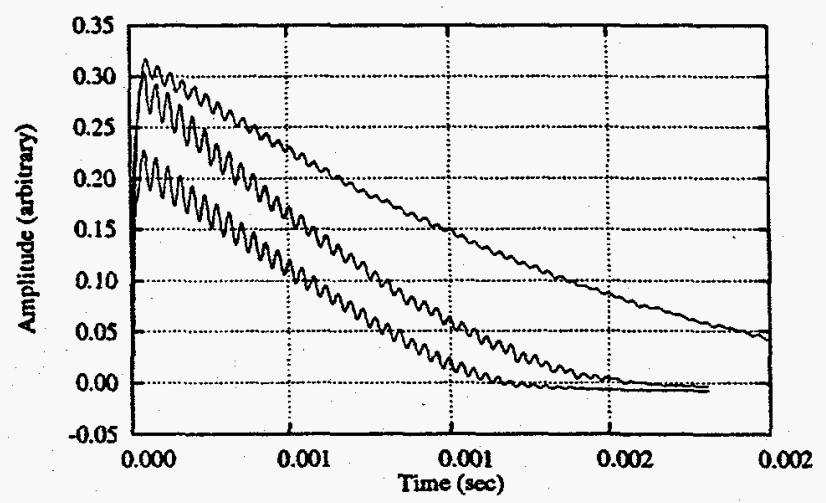

Figure 1: Typical horizontal betatron excitation amplitude of the beam centroid as a function of time. Beam current is $2.4 \mathrm{~mA}$. The upper curve is with $\xi_{x}=3.86$, the middle curve is with $\xi_{x}=6.097$, and the lower curve is with $\xi_{x}=$ 7.71 .

The damping rates as a function of the beam current and chromaticity in both horizontal and vertical planes are shown in Fig. 2 and 3. The measured vertical damping time at zero current is about $11.7 \mathrm{~ms}$ which is close to the synchrotron radiation damping time. In the horizontal plane, the damping time at zero current is $4.3 \mathrm{~ms}$. Notice that in the present operation configuration, the design synchrotron radiation damping time $\tau_{x}, \tau_{y}, \tau_{\theta}$, is $10.5,13.75$, $8.13 \mathrm{~ms}$, respectively. It shows that Landau damping effect of

DISTRIBUTION OF THIS DOCUMENT IS UNLIMITED BSIAPSTER 
is much stronger in the horisontal plane than that in the vertical plane.

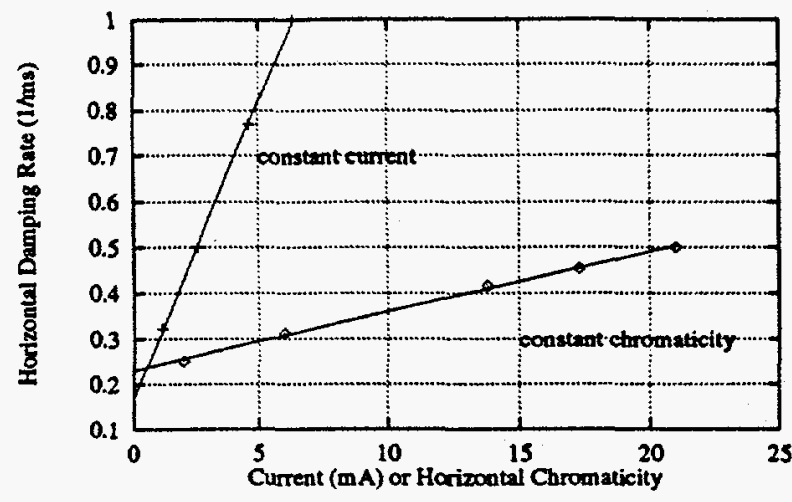

Figure 2: Horizontal damping rate as a function of the horizontal chromaticity with bunch current $2.7 \mathrm{~mA}$ or a function of bunch current at constant chromaticity.

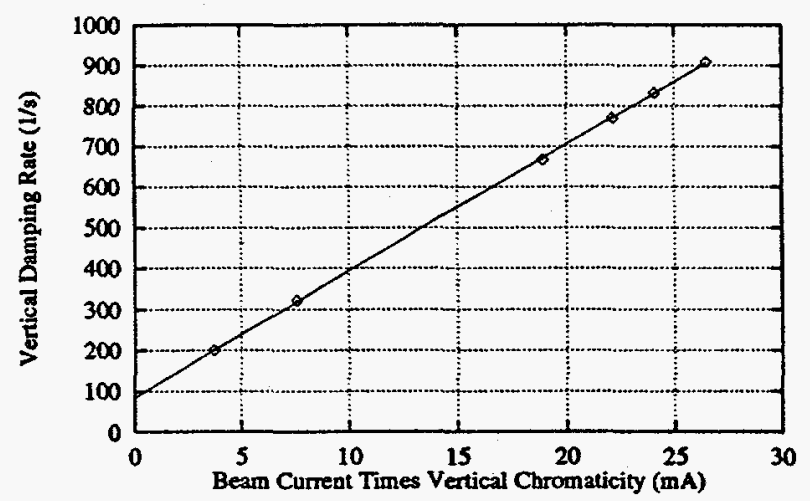

Figure 3: Vertical damping rate as a function of bunch current times vertical chromaticity.

In Fig. 1 the decoherence and recoherence modulation amplitude depends on the chromaticity and the frequency is the same as the synchrotron oscillation frequency $\nu_{1}=27$ $\mathbf{k H z}$. This is due to incoherent synchrotron oscillation. [3, $4,5]$ The modulation amplitude increases as chromaticity is larger. If the bunch purity is not good and hence there exists satellite bunch, we observed some distinct modulation frequency other than the synchrotron frequency, which differed in each plane, corresponding to the frequency beating of the betatron sidebands. The measured vertical tune shift as a function of bunch current was about $0.2 \mathrm{kHz} / \mathrm{mA}$. Hence, the modulation period becomes longer and modulation amplitude is larger as the main bunch current approaches the satellite bunch current. Fig. 4 gives an example of the modulation frequency at different current ratio in the vertical plane.

A transverse damping system is currently under development and test. The system is a wide band damping

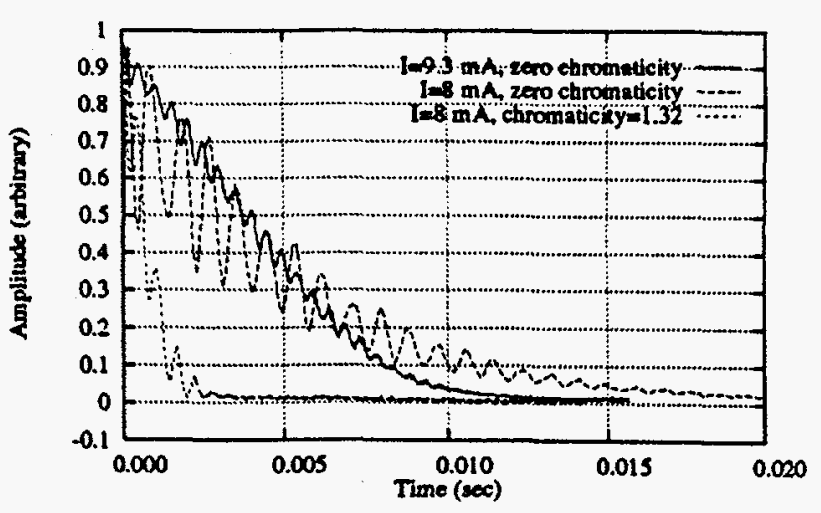

Figure 4: The decay pattern of the vertically excited beam with bunch impurity. In the beginning the beam current is $9.3 \mathrm{~mA}$ and then decay to $8 \mathrm{~mA}$. The impurity is increased because the main bunch current decay rate is fast due to Touschek scattering. The beating period is increased when impurity becomes larger. The introduction of the high chromaticity increases the decay rate of the excited beam centroid.

system. The system shall be of help in the suppression of the transverse beam oscillation in the routine operation at high current mode for the synchrotron light users. Fig. 5 depicts the damping effect with transverse damping system. The stored bunch current was not pure, therefore the distinct amplitude modulation appeared.

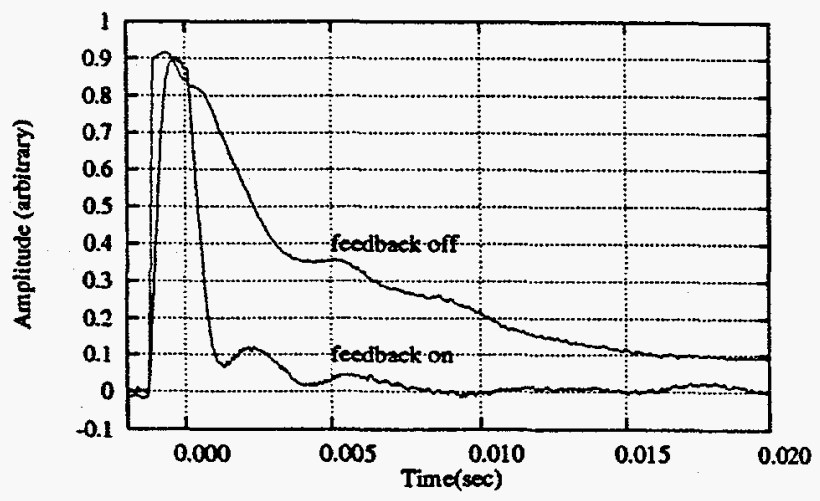

Figure 5: The damping effect of the transverse feedback system.

\section{LONGITUDINAL DAMPING}

In the longitudinal plane, we excited the beam with If frequency modulation at synchrotron frequency for a few $\mu$ and then measured the decay rate of the amplitude of the synchrotron sideband. We performed the measurements either in the single bunch mode or in a fewbunch mode. Significant decrease of the damping time in 


\section{DISCLAIMER}

Portions of this document may be illegible in electronic image products. Images are produced from the best available original document. 
the multibunch mode was observed as compared with that in the single bunch mode as shown in Fig. 6. The rea$80 \mathrm{n}$ is that the increase of synchrotron tune spread which is due to the longitudinal coupled bunch instabilities induces Landau damping. $[6,7]$ Landau damping rate is very large at high multibunch operation current and is consistent with the measured oscillation amplitude in the longitudinal plane. In order to avoid the increase of the emittance and spoiling of the undulator spectral quality, we will implement a bunch-by-bunch longitudinal damping system to realize the low emittance lattice design.

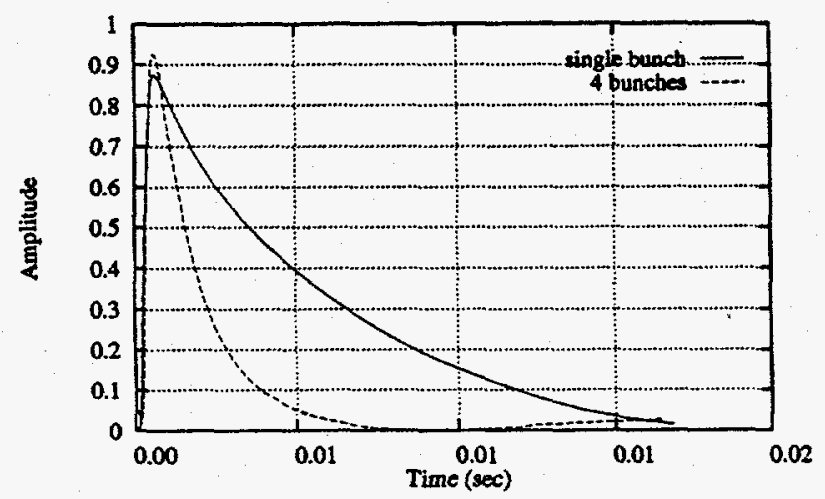

Figure 6: The decay pattern of the synchrotron sideband of the longitudinally kicked beam in the single bunch mode and in the case with four symmetrically distributed bunches. Landau damping is significant with four bunches due to longitudinal coupled bunch instabilities.

\section{ACKNOWLEDGEMENT}

The authors would like to thank H. P. Chang for his assistance in editing of this article.

\section{REFERENCES}

[1] C.C. Kuo, et. al., " Performance of the SRRC Storage Ring and Wiggler Commissioning", these proceedings.

[2] W.T. Weng et. al., "Beam Stability at SRRC Storage Ring", these proceedings.

[3] F. Willeke, "Using the Bevatron Beam Position Monitor System to Investigate Transverse Phase Space", Fermilab Report TM-1309 (1985).

[4] R.E. Meller, et. al., "Decoherence of Kicked Beams", SSL-N-360 (1987).

[5] S. R. Mane, "Tuneshifts, Tunespreads and Decoherence", FN-502 (1988).

[6] W.K. Lau, et. al., "Study of Longitudinal Coupled Bunch Instabilities in the SRRC Storage Ring", these proceedings.
[7] M.H. Wang, et. al., "The Observation of Longitudinal Coupled Bunch Motion on Streak Camera at SRRC", these proceedings.

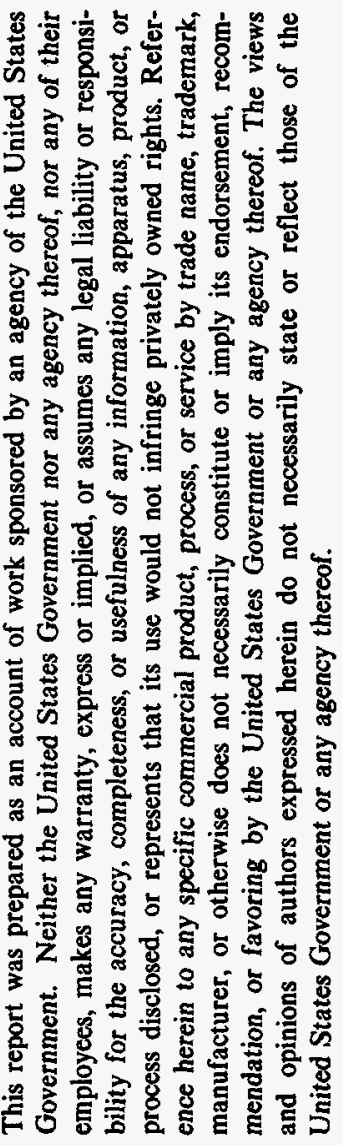

